\title{
BMJ Open The Suspected CANcer (SCAN) pathway: protocol for evaluating a new standard of care for patients with non- specific symptoms of cancer
}

Brian D Nicholson, ${ }^{1}$ Jason Oke, ${ }^{1}$ Claire Friedemann Smith, ${ }^{1}$ Julie-Ann Phillips, ${ }^{2}$ Jennifer Lee, ${ }^{2}$ Lucy Abel, ${ }^{1}$ Sadie Kelly, ${ }^{1}$ Isabella Gould, ${ }^{1}$ Toni Mackay, ${ }^{2}$ Zoe Kaveney, ${ }^{3}$ Suzie Anthony, ${ }^{2}$ Shelley Hayles, ${ }^{3}$ Daniel Lasserson, ${ }^{2,4}$ Fergus Gleeson ${ }^{2}$

To cite: Nicholson BD, Oke J, Friedemann Smith C, et al. The Suspected CANcer (SCAN) pathway: protocol for evaluating a new standard of care for patients with non-specific symptoms of cancer. BMJ Open 2018;8:e018168. doi:10.1136/ bmjopen-2017-018168

- Prepublication history and additional material for this paper are available online. To view these files, please visit the journal online (http://dx.doi. org/10.1136/bmjopen-2017018168).

Received 9 June 2017 Revised 7 November 2017 Accepted 8 November 2017

Check for updates

${ }^{1}$ Nuffield Department of Primary Care Health Sciences, University of Oxford, Oxford, UK

${ }^{2}$ Oxford University Hospitals NHS Foundation Trust, Oxford, UK

${ }^{3}$ Delivery and Localities Directorate, Oxfordshire Clinical Commissioning Group, 0xford, UK

${ }^{4}$ Nuffield Department of

Medicine, University of Oxford, Oxford, UK

Correspondence to

Dr Claire Friedemann Smith;

claire.friedemann@phc.ox.ac.uk

\section{ABSTRACT}

Introduction Cancer survival in England lags behind most European countries, due partly to lower rates of early stage diagnosis. We report the protocol for the evaluation of a multidisciplinary diagnostic centre-based pathway for the investigation of 'low-risk but not no-risk' cancer symptoms called the Suspected CANcer (SCAN) pathway. SCAN is a new standard of care being implemented in Oxfordshire; one of a number of pathways implemented during the second wave of the Accelerate, Coordinate, Evaluate (ACE) programme, an initiative which aims to improve England's cancer survival rates through establishing effective routes to early diagnosis.

Methods and analysis To evaluate SCAN, we are collating a prospective database of patients referred onto the pathway by their general practitioner (GP). Patients aged over 40 years, with non-specific symptoms such as weight loss or fatigue, who do not meet urgent cancer referral criteria or for whom symptom causation remains unclear after investigation via other existing pathways, can be referred to SCAN. SCAN provides rapid CT scanning, laboratory testing and clinic review within 2 weeks. We will follow all patients in the primary and secondary care record for at least 2 years. The data will be used to understand the diagnostic yield of the SCAN pathway in the short term (28 days) and the long term (2 years). Routinely collected primary and secondary care data from patients not referred to SCAN but with similar symptoms will also be used to evaluate SCAN. We will map the routes to diagnosis for patients referred to SCAN to assess costeffectiveness. Acceptability will be evaluated using patient and GP surveys.

Ethics and dissemination The 0xford Joint Research Office Study Classification Group has judged this to be a service evaluation and so outside of research governance. The results of this project will be disseminated by peerreviewed publication and presentation at conferences.

\section{BACKGROUND}

England's rates of cancer survival lag behind many other European countries, and late stage at diagnosis is thought to play a large

\section{Strengths and limitations of this study}

- The Suspected CANcer (SCAN) pathway will be evaluated in relation to diagnostic yield, time to diagnosis, cost-effectiveness, patient satisfaction and incidental diagnoses.

- Data from both the primary and secondary care record will be used to populate a bespoke prospective database detailing the cohort of patients evaluated by the SCAN pathway.

- A general practitioner or patient-level randomised implementation of SCAN was not feasible within the constraints of the local health system, nor a randomised stepped-wedge rollout to the six Oxfordshire Clinical Commissioning Group (CCG) regions.

- Instead, a pragmatic service evaluation is being conducted around a phased roll-out of the pathway against the previous standard of care in the same region, operated by the same CCG.

- The findings of this evaluation will only indicate SCAN's effectiveness in Oxfordshire and should not be generalised to the rest of England.

part in this. ${ }^{1}$ Twenty-one per cent of cancers are diagnosed as an emergency, which is associated with advanced tumour stage and increased mortality in the first year post-diagnosis. ${ }^{34}$ It is estimated that, if diagnosed early, 5000 cancer deaths could be prevented every year for breast, colorectal and lung cancers alone. $^{25}$ The Accelerate, Coordinate, Evaluate (ACE) programme is an early diagnosis initiative supported by the National Health Service (NHS) England, Cancer Research UK (CRUK) and Macmillan Cancer Support. ${ }^{6}$ It was formed to help improve England's cancer survival rates by generating evidence on how best to configure diagnostic pathways to drive a shift from late to early cancer at diagnosis, 
reduce the number of cancers diagnosed as an emergency and improve patient experience.

The first wave of ACE comprised around 60 projects aiming to evaluate local initiatives to develop a national body of evidence to inform cancer commissioning. ${ }^{7} \mathrm{~A}$ weakness in the current system identified during Wave 1 was the lack of a clear urgent referral pathway for patients with non-specific but concerning symptoms known to be associated with a range of cancer sites, such as fatigue, abdominal pain and weight loss. ${ }^{8}$ Consequently, before reaching a cancer diagnosis, these patients often have multiple tests and non-urgent referrals resulting in delays in diagnosis. ${ }^{9}$ The Independent Cancer Taskforce outlined the need to explore new models of care to speed up diagnosis in patients with non-specific symptoms, making references to the multidisciplinary diagnostic centre (MDC) concept. ${ }^{10}$ ACE Wave 2 was set up to facilitate the development and evaluation of a small number of MDC-based pathways in the English NHS. ${ }^{6}$ These pathways are implemented as standards of care in participating regions, in addition to site-specific urgent cancer referral pathways.

Oxfordshire's Suspected CANcer (SCAN) pathway emulates a Danish MDC pathway, the non-specific symptoms and signs of cancer patient pathway (NSSC-CPP) ${ }^{11}$ Patients first undergo a panel of diagnostic investigations including blood and urine tests and diagnostic imaging. If no diagnosis is made, but cancer or another serious disease is suspected, the patient is referred to the MDC. ${ }^{11} 12$ The MDC is a diagnostic unit with access to a broad range of investigations and specialist expertise in managing patients with non-specific symptoms. Of 1278 patients referred to the NSSC-CPP pathway by their general practitioners (GPs), a cross-sectional study reported that $16 \%$ of patients were diagnosed with cancer. ${ }^{13}$ The most common symptoms recorded were weight loss $(53 \%)$, fatigue $(50 \%)$ and pain $(37 \%)$. The most common clinical findings were 'affected general condition' (36\%), GP 'gut feeling' (23\%) and abnormal abdominal examination (13\%). Forty-eight per cent of patients were referred with abnormal blood test results. Cancer was diagnosed across a broad range of 18 subgroups, the most common of which were lung (18\%), colorectal $(13 \%)$, haematological $(10 \%)$, pancreatic $(9 \%)$ and upper gastrointestinal $(8 \%) .{ }^{13}$ A later cohort study including 938 patients referred to the NSSC-CPP reported that $35 \%$ were diagnosed with serious disease within 3 months, of which one-third had cancer. ${ }^{14}$

As is the case in the UK, healthcare in Denmark is mostly free to access for residents, and Danish GPs act as 'gatekeepers' to specialist services. ${ }^{15}$ Five-year survival rates for several cancer types are also among the lowest in Organisation for Economic Cooperation and Development countries. ${ }^{15}$ Both countries have introduced a 1-month standard for the time between referral and diagnosis to increase the proportion of cancers diagnosed at an early stage. ${ }^{1516}$ Furthermore, following the introduction of cancer pathways in Denmark which incorporate patient review by multidisciplinary teams (MDTs), waiting times have significantly reduced across almost all cancer types. ${ }^{17}$ The similarities between the Danish and UK health systems, the challenges faced by both and the improvements brought about by MDC-based pathways suggest that these pathways for non-specific symptoms warrant evaluation in the UK. At the time of writing, we retrieved no peer-reviewed articles detailing MDC pathways for cancer diagnosis in the UK, and only one conference abstract describing 91 patients assessed via an alternative MDC pathway developed during ACE Wave 1 in London. ${ }^{18}$ Robust evaluation of the SCAN pathway has the potential to contribute to the evidence base for the MDC concept in cancer diagnosis. We report here the protocol for the SCAN pathway evaluation.

\section{AIM}

The aim of this study is to evaluate the SCAN pathway, a new standard of care for the rapid investigation of patients with non-specific cancer symptoms in Oxfordshire. SCAN will be evaluated in terms of how well it meets its objectives, which are detailed below.

\section{Objectives}

In line with the CRUK ACE initiative which aims to reduce late and increase early cancer diagnosis, decrease cancer diagnoses made through emergency presentations and improve patient experience, ${ }^{6}$ the objectives of the SCAN pathway are to:

- reduce time from initial primary care presentation with symptoms to diagnosis

- achieve a higher proportion of early stage cancer at diagnosis

- improve patient experience of the diagnostic pathway

- establish whether the MDC model is cost-effective.

\section{Setting}

Oxfordshire's cancer incidence (600 cases per 100000 ) is lower than the UK average (615 cases per 100000$)$. Cancer mortality (261 per 100000 ) is also lower than the national average, with fewer cancers diagnosed through emergency presentation (17.1\% vs $20.1 \%)$ and more patients diagnosed at an early stage $(56.8 \%$ vs $54.3 \%)$ than the national average. ${ }^{19}$ Comprising a predominantly white $(90.85 \%)$ population, Oxfordshire has smaller foci of Asian (4.84\%), Black (1.75\%) and mixed ethnic $(2.02 \%)$ groups. Black and minority ethnic communities form $22.4 \%$ of Oxford city's population, with lower proportions in more rural districts: $7.8 \%$ in Cherwell and $3.2 \%$ in West Oxfordshire (source: 2011 Census $^{20}$ ). Rural districts $(67 \%)$ rank in the $10 \%$ least deprived, and urban $(33 \%)$ in the $20 \%$ most deprived in England. There are no ACE Wave 1 sites in Oxfordshire.

The Oxfordshire Clinical Commissioning Group (CCG) serves a population of over 700000 through 70 general practices. ${ }^{21}$ Oxford University Hospitals NHS Foundation Trust (OUHFT) is made up of four hospitals providing 
a range of specialist services (John Radcliffe Hospital, Churchill Hospital, Nuffield Orthopaedic Centre and the Horton General Hospital). SCAN imaging takes place at the Churchill Hospital, and the SCAN MDC is located at the John Radcliffe Hospital. SCAN links with the Oxford Allied Health Science Network (AHSN) imaging network, aiming to develop a model for expansion through the seven adjoining NHS network trusts.

\section{The SCAN pathway}

SCAN retains the GP's gate-keeping role, requiring patients to first attend their GP with symptoms to access the pathway through GP referral. ${ }^{22}$ The SCAN referral algorithm was developed by consensus between an MDT including GPs, radiologists, physicians and health service researchers (online supplementary appendix 1). It incorporates age-thresholds and 'low-risk but not no-risk' symptoms that fall outside of existing urgent 2-week-wait (2ww) referral pathways based on the National Institute for Health and Care Excellence NG12 suspected cancer guidelines ${ }^{23}$ but remain predictive of cancer in primary care.

SCAN will be opened up sequentially to GPs in each of the six subregions of Oxfordshire CCG, to ensure that the service has enough capacity to meet demand. The first region opened on 15 March 2017 and the second on 5 June 2017. In response to demand, regions three and four were opened on 6 September 2017, and the final two regions are expected to open by 9 November 2017 .

\section{Estimated referral rate}

We used the following data to estimate expected referral rates: (1) Oxfordshire population statistics ${ }^{20}$; (2) the number of GPs in Oxfordshire ${ }^{21}$; (3) the referral rate reported for the Danish NSSC-CPP pathway ${ }^{12}$; (4) the estimated prevalence of non-specific symptoms meeting SCAN referral criteria in primary care populations, derived from the control groups of primary care-based case-control studies. ${ }^{24}$ Using these sources, an estimate of 20-40 referrals per week was anticipated, taking into account that not all patients presenting to primary care with qualifying symptoms will be referred by their GP: symptoms may not occur in isolation or a pre-existing condition will provide explanation; GPs may identify an alternative explanation for new symptoms negating the need for referral; patients may be referred by other routes or patients may decline referral.

\section{Referral criteria}

A structured standardised electronic referral form has been disseminated to all GPs in Oxfordshire (online supplementary appendix 2). If there is no other urgent referral pathway for the clinical scenario, patients aged $\geq 40$ years are accepted if their GP is concerned about cancer or serious disease following face-to-face primary care assessment of individual or combined 'low-risk but not no-risk' symptoms. In addition, patients may be referred based on their GP's clinical suspicion of cancer or serious disease (their 'gut feeling'). ${ }^{25}{ }^{26}$ GPs are also requested to indicate their suspicion of malignancy at this stage. The essential referral criteria are:

1. There is no other urgent referral pathway suitable for this clinical scenario.

2. Patient is $\geq 40$ years of age

3. Patient has at least one of the following:

- Unexplained weight loss

- Severe unexplained fatigue

- Persistent nausea or appetite loss

- New atypical pain

- Unexplained laboratory test finding

- GP clinical suspicion of cancer or serious disease/GP 'gut feeling'

\section{Stages of the pathway}

As part of their initial work-up to exclude more common causes of non-specific symptoms in primary care, GPs will conduct investigations essential to allow access to the SCAN pathway: creatinine (necessary prior to intravenous contrast) and thyroid function tests (hypothyroidism as a cause of fatigue). The referral form completed by the GP constitutes a referral for all aspects of the SCAN pathway, including the CT scan and blood tests. Once referred, a member of the SCAN team (the SCAN pathway navigator) assumes clinical responsibility for the patient, confirms that the patient meets the inclusion criteria, orders and coordinates the CT and blood tests, and provides a point of contact for the patient. Demographic and clinical information is captured by the GP referral form.

Consent: At the point of referral, patients are given a participant information sheet detailing the SCAN pathway and consent form (online supplementary material 3) to allow their anonymised medical records to be used for the purposes of evaluating the pathway. Patients are given time to take the information away and consider whether they wish to participate. If patients decide to participate, they are asked to take the consent form to the initial appointment, at which time they may ask any outstanding questions. Patients who do not wish to have their medical records used may still be referred onto the SCAN pathway but will not be followed up for the purposes of the evaluation. At the time of writing, none of the patients accepted onto SCAN had refused consent.

Stage 1: GP direct access triage tests: At the first hospital appointment, a panel of standard diagnostic investigations with rapid turnaround (request-test-report) of $<7$ days are performed. These tests include a panel of blood tests, faecal immunochemical testing and appropriate low-dose CT imaging with separate reporting lines to ensure report turnaround times of 24 hours (box). Separate reporting lines for radiology facilitate evaluation, and the AHSN imaging consultants provide additional reporting capacity to ensure turnaround times.

Stage 2: The clinical information obtained in stage 1 directs the patient's subsequent progression through the pathway. Patients are either referred: 


\section{Box Tests performed at SCAN stage 1}

- Full blood count

- Erythrocyte sedimentation rate

- C reactive protein

- Urea and electrolytes

- Creatinine

- Calcium

- Phosphate

- HbA1c

- Thyroid function

- CA125 (females)

- PSA (males)

- Faecal immunochemical testing

- CT (thorax, abdomen and pelvis)

CA125, cancer antigen 125; HbA1c, glycated haemoglobin; PSA, prostate-specific antigen; SCAN, Suspected CANcer.

a. to a cancer MDT or specialist clinic via an existing urgent pathway

b. for additional direct access investigation within 1 week prior to clinician review

c. to the MDC for medical review.

The referring GP receives a copy of the CT report and a letter from the SCAN team explaining which pathway their patient has been referred to. Any urgent findings are communicated to the GP via telephone. Any telephone conversations are followed by a letter detailing the conversation and the actions the GP and SCAN team have agreed to.

Stage 3: If symptom causation remains unclear after 2a or $2 \mathrm{~b}$, the patient is automatically referred to the MDC (2c). At the point of referral to the MDC, the accepting hospital clinician becomes the responsible MDC clinician. At the MDC, the sequence of testing to further explain the patient's clinical problem is determined by the accepting clinician.

Stage 4: All patients who consent to participate in the SCAN pathway will be followed up for 2 years, including patients for whom cancer and serious disease is excluded. The GP will receive a structured follow-up plan allowing return to the MDC to avoid repeat CT scanning for patients with new, recurrent or persistent symptoms meeting SCAN entry criteria. The patient journey along the SCAN pathway is summarised in online supplementary appendix 4 .

\section{METHODS FOR EVALUATION}

The sequential implementation of the SCAN pathway in Oxfordshire has allowed a short informal 'pilot' period during which we have been able to assess the functioning of the pathway to address any issues that may arise before it is opened to all GPs. A detailed analysis of the consecutive cohort of patients referred to the SCAN pathway, whose medical records are gathered retrospectively and followed up prospectively, will form the basis of the pathway's evaluation. We will also evaluate the pathway in terms of its cost-effectiveness as well as a number of other indicators detailed below, thereby following the framework for evaluating complex interventions as laid out by the Medical Research Council. ${ }^{27}$

\section{SCAN database}

The OpenClinica computer package (https://www. openclinica.com/) is being used to store a database of demographic information, symptoms leading to referral, investigations performed, referrals made, appointments, diagnoses and short-term and long-term outcomes for all patients referred to SCAN. The time-point and outcome of each clinical encounter will be recorded for at least 2 years following referral to identify short-term and longterm diagnoses.

Patients will enter the database on the date the SCAN team confirms their eligibility. At this point, data collected retrospectively from the primary care record using the autopopulating referral form (online supplementary appendix 2) will be manually entered into the SCAN database, and prospective data collection will begin using the OUHFT record.

The SCAN pathway will be evaluated based on its shortterm and long-term diagnostic yield and its cost-effectiveness in terms of the resources and time needed for a diagnosis to be reached. Patients' route to diagnosis and satisfaction with their experience of the SCAN pathway will also be evaluated as secondary outcomes. The primary and secondary points of evaluation are described in detail below.

\section{Primary points of evaluation}

Diagnostic yield

a. Short-term: diagnoses made within 28 days of referral.

b. Cancer site and stage at diagnosis: histopathology or MDT determined.

c. Non-cancer diagnoses determined by MDC or another specialist clinic.

d. Long-term: diagnoses made within 2 years of referral confirmed by primary and secondary care database review at 2 years.

\section{Secondary points of evaluation}

a. To map the route to diagnosis for SCAN patients in terms of time intervals associated with diagnosis (ie, each diagnostic interval in line with the Aarhus statement $\mathrm{t}^{28}$ ) and the number and sequence of patient encounters (investigations and appointments leading to diagnosis), including the number of diagnoses made following an emergency presentation.

b. To quantify incidental findings detected by the SCAN pathway.

c. To evaluate the cost-effectiveness of the SCAN pathway.

d. To assess patient and GP satisfaction with the SCAN pathway. 


\section{SCAN implementation}

The implementation of the SCAN pathway will be carried out in six stages corresponding to the six Oxfordshire CCG subregions. This pragmatic decision was made to allow the OUHFT and CCG to monitor GP uptake of the pathway in real-time to ensure that the capacity of the pathway is not exceeded and to allow early problems with service delivery to be overcome. This is an opportunity for a rigorous evaluation of the pathway in real-time, in the same county and which will avoid the potential confounding that could arise from comparing SCAN patients to patients in different regions operated by different CCGs.

Pre-SCAN period: Before having access to SCAN, GPs in each region will be asked to prospectively identify patients meeting SCAN entry criteria and to complete a 'dummy' comparator cohort data collection form to be submitted by email to a secure CCG email inbox (online supplementary appendix 5). Anonymised referral information is extracted electronically to maintain patient confidentiality. This group of comparator patients will provide data about patients receiving the standard of care prior to the introduction of SCAN (the new standard of care) who are diagnosed via alternative routes, such as $2 \mathrm{ww}$ pathways, non-urgent specialist referral or by emergency presentation. In addition, an audit of local GP electronic records of patients with symptoms meeting SCAN referral criteria, but referred via other routes for investigation, will provide further contemporaneous data against which to evaluate SCAN.

SCAN period: Depending on uptake of the pathway, each CCG region will transition to the SCAN pathway over time. The set-up and evaluation of the SCAN pathway is currently funded for a period of at least 2 years of patient intake and 2 years of follow-up. ACE Wave 2 projects are expected to report results in late 2018. ${ }^{6}$ Following this, it is anticipated that Oxfordshire CCG will adopt SCAN indefinitely and will take on its funding as the MDC model is of interest to NHS England.

Follow-up: All patients will be followed up in the primary and secondary care record for at least 2 years from entry by the pathway navigators.

\section{STATISTICAL ANALYSIS}

\section{Primary point of evaluation: diagnostic yield}

Our primary point of evaluation is yield from the new SCAN pathway with respect to the number and proportion of patients (1) with a new diagnosis or with disease excluded within 28 days and (2) with a diagnosis made within 2 years of referral. For newly diagnosed patients, we will report the diagnostic interval (first presentation to primary care to date of diagnosis), the doctor interval (first presentation to primary care to the first primary care investigation), the primary care interval (first presentation to primary care to referral to secondary care), secondary care interval (referral to secondary care to start of treatment) and the treatment interval (diagnosis to the start of treatment).$^{28}$ For patients with indeterminate findings which require further investigation, we will report the number of follow-up consultations or investigations up until discharge.

The analysis of long-term outcomes will be conducted after 2 years, where missed diagnoses (false negatives) will be defined as diagnoses not picked up in the short term but in the longer term and attributed to the initial symptomatic presentation allowing entry to the cohort. Within this analysis, the proportion of patients diagnosed with cancer and surviving 1 year will be ascertained.

\section{Secondary points of evaluation: route to diagnosis, cost- effectiveness, GP and patient satisfaction}

For the secondary outcome assessing routes to diagnosis, the number (and type) of healthcare contacts required to make a diagnosis will be counted for each participant starting from the initial primary care visit for the symptoms permitting referral to SCAN. The number of patients diagnosed following an emergency presentation will also be counted. Medians and IQRs will be calculated for each of the diagnostic intervals (days) stratified by symptom group, disease type, disease site and severity/stage where possible and presented graphically using box plots.

Incidental findings: Incidental findings are radiological abnormalities not caused by the symptoms being investigated that may drive further imaging and concern. ${ }^{29}$ To understand incidental findings in patients referred to SCAN, demographic, clinical and radiological information will be extracted from the SCAN database. Imaging findings will be categorised per anatomical location. Each finding will be defined as of potential clinical importance (eg, cancer, aneurysms and cardiac findings), and probable or consistent with, or equivocal or unlikely to explain the symptoms at the time of referral. In the case of multiple lesions of the same type, the number will be recorded and reported. As the SCAN pathway uses ungated low-dose CT imaging, an approach to avoid the over interpretation of cardiac findings was developed. The TeraRecon software package (https://www.terarecon.com/) will be used to look at any coronary artery calcification, and an Agatson score will be calculated. This approach has been adopted due to the success that has been reported in a number of American studies assessing the prognostic accuracy of calcium scoring coronary arteries from an ungated low-dose CT scan. ${ }^{30-34}$ The data gathered from patients' medical records will be used to evaluate the ability of this protocol to show relevant coronary artery calcification. Incidental findings that have potential clinical importance will be followed up according to the standards issued by the American College of Radiologists, the British Thoracic Society and the Royal College of Radiologists. ${ }^{35-37}$ In addition, any further investigations (eg, MRI) required to determine if CT findings are truly incidental will be recorded as part of mapping routes to diagnosis.

Patient survey: All patients who consent to participate in the evaluation of the SCAN pathway will be asked to complete a set of questionnaires about their experience 
of the pathway. The Consequence of Screening (COS) questionnaire (originally Psychological Consequences Questionnaire) ${ }^{38}$ will be given to patients shortly after referral to the MDC and then again 6-12months after their referral. Individual items on the COS scales will be combined into themes of anxiety, behaviour, sense of dejection and sleep, and item scores added together. COS domain scores will be compared across patients who had a confirmed diagnosis of cancer or other serious disease, false positive finding and probably benign finding using non-parametric tests.

Patient satisfaction surveys will also be distributed to all patients (regardless of participation in the pathway evaluation) at the end of their CT scan. This questionnaire has been designed to evaluate the patient's experience of the staff and the service provided by the radiology department.

GP satisfaction survey: A brief satisfaction survey will be distributed to all participating GPs. This survey will assess satisfaction with the ease of use of the SCAN pathway, speed with which referred patients are seen by the SCAN team and the quality of the information provided to GPs by the SCAN team both in terms of the functioning and purpose of the pathway and the results of the diagnostic tests undergone by their patient(s). The survey will be distributed and completed online using the Bristol Online Surveys tool (https://www. onlinesurveys.ac.uk/).

Cost-effectiveness: The cost-effectiveness of the SCAN pathway will be assessed from the perspective of the NHS in England, using a within-trial analysis. Each patient encounter from referral to SCAN up until a final diagnosis is made or excluded will be recorded. The outcome will be incremental cost-effectiveness compared with the pre-SCAN pathway, with effectiveness measured in unit reduction in time to diagnosis, and in additional diagnosis within 28 days. The comparison group will be the pre-SCAN patient cohort. Although the numbers of comparator patients should closely resemble those of patients referred to the pathway as the inclusion criteria are identical, we expect comparator patient numbers to be lower as GPs are requested to take time to complete the referral forms with no tangible benefit to their patients. Due to the expected lower response rate, we will supplement comparator data from GPs with an audit of Oxfordshire surgeries with the appropriate data sharing agreement, approximately 60 practices, up to March 2017. The resource use of patients in the SCAN pathway and its comparator will be estimated from the database and costed using national unit costing databases. ${ }^{39}$ In addition, patients will be asked to complete the UK Cancer Costs Questionnaire ${ }^{40}$ to record ongoing financial and opportunity costs. Sensitivity analyses will be carried out on the key assumptions of the evaluation, including resource use assumptions, the impact of adjusting for baseline characteristics and extending the analysis to cost utility.

\section{Eligible patients not referred to SCAN}

The phased introduction of the SCAN pathway affords comparison with outcomes under the previous standard of care for patients meeting SCAN referral criteria. This will include patients with SCAN eligible symptoms identified by their GPs and diagnosed via alternative routes, for example, by $2 \mathrm{ww}$ pathways, non-urgent specialist referral or by emergency presentation. Robust statistical comparisons between SCAN and the period prior to SCAN may be limited dependent on gathering data on a sufficient number of patients with symptoms meeting SCAN referral criteria who are not referred to SCAN, which is in turn dependent on GPs completing the comparator referral forms. Therefore, the data collected on patients not referred to SCAN provided by GPs will be supplemented with an audit of the primary care record and will be presented in tables with descriptive statistics, only comparing SCAN and pre-SCAN outcomes when appropriate.

\section{Sample size}

In order to have power $>80 \%($ alpha $=5 \%)$ and if the SCAN pathway halves the average length of the diagnostic interval compared with usual care $(\mathrm{HR}=2)$, we would need at least 43 patients diagnosed with cancer during the pre-SCAN period and at least 173 patients diagnosed with cancer following the introduction of the pathway. ${ }^{41}$ If $15 \%$ of patients presenting to their GP with non-specific symptoms have cancer, ${ }^{12}$ we need to recruit at least 1460 patients.

\section{Data handling and data management}

A data management plan (DMP) is in place outlining in detail the specific procedures to ensure that high-quality data are produced for statistical analysis. The DMP was reviewed and signed off by all relevant parties prior to data management activities commencing.

Data will be collected electronically in OpenClinica (https://www.openclinica.com/), and data validation is achieved through electronic programmed checks or through manual review of listing outputs. All discrepancies generated by electronic validation checks or manual listings will be reviewed by the data manager.

\section{ETHICS AND DISSEMINATION}

Evaluation of a newly established service/adopted pathway does not require research governance as such activity falls outside of the definition of research as set out by the Health Research Authority (HRA) and would not be considered research in the NHS. As such, this evaluation is not subject to the Department of Health's Research Governance Framework for Health and Social Care (2005). This opinion can be reviewed by reference to the HRA's algorithm, available at http://www.hradecisiontools.org.uk/research/ and attendant leaflet, Defining Research or by reference to the Healthcare Quality Improvement Partnership's Guide for Clinical 
Audit, Research and Service Review. ${ }^{42}$ In addition, a Privacy Impact Assessment concluded that individual patient consent was not necessary to collect data on the comparator patients. The primary reason for this was that obtaining consent was likely to cause unnecessary distress to patients who were not yet able to make use of the new pathway. Instead, a data sharing agreement was signed by all participating GP surgeries, and all comparator data will be sent electronically to the CCG Commissioning Support Unit and pseudonymised before being shared with the SCAN team.

The results of this evaluation will be reported in peer-reviewed journals and on the websites of the various ACE Wave 2 funding bodies. Abstracts for oral or poster presentations will be submitted to national and international conferences. Data resulting from this study will be made available following a request to the authors.

\section{Strengths and limitations}

This is the first published protocol for the evaluation of an MDC pathway based in the UK. The evaluation outlined above will provide detailed information on the diagnostic yield and time to diagnosis for comparison of the MDC model with existing routes to diagnosis in the NHS. Our focus on incidental findings and cost-effectiveness will add valuable evidence about the value of early CT scanning of patients with non-specific symptoms, especially in response to concerns about overdiagnosis.

Our evaluation, however, has some limitations. First, due to the pragmatic nature of SCAN's implementation, the evaluation has had to take into account resource constraints and logistical realities of Oxfordshire's health system. Consequently, we have been unable to randomise individual patients or GPs to the SCAN pathway, nor could we randomise the sequence of a stepped-wedge roll-out of SCAN across CCG regions. Second, collection of comparator data is reliant on local GPs opting to do so as an additional task in an already busy health service without additional financial incentive. We are supplementing this approach with a retrospective review of primary care records to identify eligible patients pre-SCAN. The method of symptom capture using this approach is in development but may be limited to retrospective electronic-coded entries. Due to these factors, the generalisability of our findings outside of Oxfordshire will be limited.

\section{CONCLUSION}

Cancer prognosis improves with early diagnosis, but the UK lags behind many European countries in terms of the proportion of patients diagnosed at an early stage. For this reason, the Independent Cancer Taskforce has highlighted the need for alternative routes to diagnosis to be explored and has made specific reference to MDC-based pathways. The SCAN pathway is such an MDC-based pathway which has been adopted in Oxfordshire with the aim of reducing the time from initial presentation of non-specific but concerning symptoms to diagnosis and increasing the proportion of cancers diagnosed at an early stage. We will evaluate the ability of the SCAN pathway to meet these aims over 2 years, will assess the patient experience of the diagnostic pathway and appraise the cost-effectiveness of the pathway.

Acknowledgements The authors would like to thank the following colleagues for their input: Karen Fitzgerald, Daniel Forrester, Richard Hobbs, Shahista Hussain, Mads Ingeman, Rachael Lewis, Karen Melham, Esben Naeser, Tom Nichols, Brenda Shanahan, Ann Van den Bruel and Professor Peter Vedsted.

Collaborators The authors would like to thank Shahista Hussain from OUHFT Research and Development who assisted with the development of the patient information leaflet.

Contributors FG, SH, SA, BDN, TM, ZK and DL designed the clinical pathway. BDN, J0 and LA designed this pathway evaluation. BDN developed the patient information leaflet. JL, J-AP, JO and SH reviewed the patient information leaflet. BDN, CFS, IG and SK designed and oversaw the building of the SCAN database. $\mathrm{BDN}, \mathrm{CFS}$ and ZK designed and set up the procedures for the audit of clinical records for comparator data. BDN, J0 and CFS wrote this protocol. All authors reviewed and gave comments, will be involved in the running of this evaluation study and the analysis of the results and have read and approved this protocol.

Funding This work is supported by Cancer Research UK, Macmillan Cancer Support, NHS England and the Department of Health's Policy Research Units. As part of the ACE programme, CRUK put out a search brief and specified some areas on which the pathways should be evaluated.

\section{Competing interests None declared.}

Patient consent Detail has been removed from this case description/these case descriptions to ensure anonymity. The editors and reviewers have seen the detailed information available and are satisfied that the information backs up the case the authors are making.

Provenance and peer review Not commissioned; externally peer reviewed.

Open Access This is an Open Access article distributed in accordance with the Creative Commons Attribution Non Commercial (CC BY-NC 4.0) license, which permits others to distribute, remix, adapt, build upon this work non-commercially, and license their derivative works on different terms, provided the original work is properly cited and the use is non-commercial. See: http://creativecommons.org/ licenses/by-nc/4.0/

(c) Article author(s) (or their employer(s) unless otherwise stated in the text of the article) 2018. All rights reserved. No commercial use is permitted unless otherwise expressly granted.

\section{REFERENCES}

1. De Angelis R, Sant M, Coleman MP, et al. Cancer survival in Europe 1999-2007 by country and age: results of EUROCARE--5-a population-based study. Lancet Oncol 2014;15:23-34.

2. Richards MA. The size of the prize for earlier diagnosis of cancer in England. Br J Cancer 2009;101(Suppl 2):S125-S129.

3. McPhail S, Elliss-Brookes L, Shelton J, et al. Emergency presentation of cancer and short-term mortality. Br J Cancer 2013;109:2027-34.

4. Cancer Research UK. Cancer diagnosis and treatment statistics. 2016 http://www.cancerresearchuk.org/health-professional/cancerstatistics/diagnosis-and-treatment\#heading-Zero (accessed 13 July 2016).

5. Abdel-Rahman M, Stockton D, Rachet B, et al. What if cancer survival in Britain were the same as in Europe: how many deaths are avoidable? Br J Cancer 2009;101(Suppl 2):S115-S124.

6. Cancer Research UK. Accelerate, Coordinate, Evaluate (ACE) programme. 2017 http://www.cancerresearchuk.org/healthprofessional/early-diagnosis-activities/ace-programme

7. Cancer Research UK. Accelerate, Coordinate, Evaluate ACE programme projects. 2016 http://www.cancerresearchuk.org/ health-professional/early-diagnosis-activities/ace-programme/aceprogramme-projects

8. Lewis R. NHS England, Cancer Research UK and Macmillan Cancer Support. Improving Diagnostic Pathways for Patients with Vague Symptoms. 2017.

9. Lyratzopoulos G, Wardle J, Rubin G. Rethinking diagnostic delay in cancer: how difficult is the diagnosis? BMJ 2014;349:g7400. 
10. Taskforce TIC. Achieving world-class cancer outcomes 2015-2020. a strategy for England. 2015.

11. Vedsted P, Olesen F. A differentiated approach to referrals from general practice to support early cancer diagnosis - the Danish three-legged strategy. Br J Cancer 2015;112 (Suppl 1):S65-S69.

12. Ingeman ML, Christensen MB, Bro F, et al. The danish cancer pathway for patients with serious non-specific symptoms and signs of cancer-a cross-sectional study of patient characteristics and cancer probability. BMC Cancer 2015;15:421.

13. Ingeman ML, Ormstrup TE, Vedsted P. Direct-access to abdominal ultrasonic investigation from general practice - the role in earlier cancer diagnosis. Fam Pract 2015;32:205-10.

14. Næser E, Fredberg $U$, Møller $\mathrm{H}$, et al. Clinical characteristics and risk of serious disease in patients referred to a diagnostic centre: A cohort study. Cancer Epidemiol 2017;50:158-65.

15. Policies European Observatory on Health Systems and Policies. The health systems and policy monitor. $2012 \mathrm{http}: / / \mathrm{hspm} .0 \mathrm{rg} /$ searchandcompare.aspx

16. Department of Health. From 2020, people with suspected cancer will be diagnosed faster 2015. https://www.gov.uk/government/ news/from-2020-people-with-suspected-cancer-will-be-diagnosedfaster

17. Probst HB, Hussain ZB, Andersen O. Cancer patient pathways in Denmark as a joint effort between bureaucrats, health professionals and politicians--a national Danish project. Health Policy 2012;105:65-70.

18. Chung D, Matoo K, Seward E, et al. PWE-131 The first uk multidisciplinary diagnostic centre: a novel cancer diagnostic service. Gut 2016;65(Suppl 1):A202.2-A203.

19. Cancer Research UK. Local cancer statistics 2016. http://www. cancerresearchuk.org/cancer-info/cancerstats/local-cancerstatistics/?location-name-1=NHS\%200xfordshire\%20CCG\& location $-1=10 \mathrm{Q}$

20. Office for National Statistics. 2011 Census. 2011 https://www.ons. gov.uk/census/2011census

21. Oxfordshire Clinical Commissioning Group. NHS Oxfordshire clinical commissioning group: annual report 2015/16.

22. Vedsted $P$, Olesen $F$. Are the serious problems in cancer survival partly rooted in gatekeeper principles? An ecologic study. B J of Gen Pract 2011;61:508-12.

23. Excellence National Institute for Health and Care Excellence: Clinical Guidelines. Suspected cancer: recognition and referral [NG12]. NICE 2015.

24. Hamilton W. The CAPER studies: five case-control studies aimed at identifying and quantifying the risk of cancer in symptomatic primary care patients. Br J Cancer 2009;101(Suppl 2):S80-S86.

25. Donker GA, Wiersma E, van der Hoek L, et al. Determinants of general practitioner's cancer-related gut feelings-a prospective cohort study. BMJ Open 2016;6:e012511.

26. Hjertholm P, Moth G, Ingeman ML, et al. Predictive values of GPs' suspicion of serious disease: a population-based follow-up study. $\mathrm{Br}$ J Gen Pract 2014;64:e346-e353.
27. Craig P, Dieppe P, Macintyre S, et al. Developing and Evaluating Complex Interventions: New guidance. Medical Research Council 2006.

28. Weller D, Vedsted P, Rubin G, et al. The Aarhus statement: improving design and reporting of studies on early cancer diagnosis. $\mathrm{Br} \mathrm{J}$ Cancer 2012;106:1262-7.

29. Raymond J, Long H, Roy DJ. Overdiagnosis, incidental findings and Socrates' three sieves. J Neuroradiol 2017;44:173-4.

30. Shemesh J, Henschke Cl, Shaham D, et al. Ordinal scoring of coronary artery calcifications on low-dose CT scans of the chest is predictive of death from cardiovascular disease. Radiology 2010;257:541-8.

31. Budoff MJ, Nasir K, Kinney GL, et al. Coronary artery and thoracic calcium on noncontrast thoracic CT scans: comparison of ungated and gated examinations in patients from the COPD Gene cohort. J Cardiovasc Comput Tomogr 2011;5:113-8.

32. Shemesh J, Henschke CI, Farooqi A, et al. Frequency of coronary artery calcification on low-dose computed tomography screening for lung cancer. Clin Imaging 2006;30:181-5.

33. Takx RA, de Jong PA, Leiner T, et al. Automated coronary artery calcification scoring in non-gated chest CT: agreement and reliability. PLoS One 2014;9:e91239.

34. Wu MT, Yang P, Huang YL, et al. Coronary arterial calcification on low-dose ungated MDCT for lung cancer screening: concordance study with dedicated cardiac CT. AJR Am J Roentgenol 2008;190:923-8.

35. Berland LL, Silverman SG, Gore RM, et al. Managing incidental findings on abdominal CT: white paper of the ACR incidental findings committee. J Am Coll Radiol 2010;7:754-73.

36. Callister ME, Baldwin DR, Akram AR, et al. British thoracic society guidelines for the investigation and management of pulmonary nodules. Thorax 2015;70:ii1-ii54.

37. Public Health England. Abdominal aortic aneurysm screening 2015. https://www.gov.uk/government/uploads/system/uploads/ attachment_data/file/493976/AAAS_-_revision_1.pdf (accessed Aug 2015).

38. Cockburn J, De Luise T, Hurley S, et al. Development and validation of the PCQ: a questionnaire to measure the psychological consequences of screening mammography. Soc Sci Med 1992;34:1129-34.

39. Curtis LaB A. Unit costs of health and social care. Canterbury: University of Kent, Unit PSSR, 2016.

40. Marti J, Hall PS, Hamilton P, et al. The economic burden of cancer in the UK: a study of survivors treated with curative intent. Psychooncology 2016;25:77-83.

41. Neal RD, Tharmanathan P, France B, et al. Is increased time to diagnosis and treatment in symptomatic cancer associated with poorer outcomes? Systematic review. Br J Cancer 2015;112 (Suppl 1):S92-S107.

42. Brain JS, Gerrish J, Mawson K, et al. A guide for clinical audit, research and service review - an educational toolkit designed to help staff differentiate between clinical audit, research and service review activities. Health and Quality Impro Partnership 2011. 